Article

\title{
Double Biocatalysis Signal Amplification Glucose Biosensor Based on Porous Graphene
}

\author{
Yaping $\mathrm{He}^{1, *}$ (D) , Jianbin Zheng ${ }^{2, *}$, Bini Wang ${ }^{3}$ and Hongjiang Ren ${ }^{1}$ \\ School of Chemical Engineering, Xi'an University, Xi'an 710065, Shaanxi, China; hjren@xawl.edu.cn \\ Institute of Analytical Science/Shaanxi Provincial Key Laboratory of Electroanalytical Chemistry, \\ Northwest University, Xi'an 710069, Shaanxi, China \\ 3 College of Food Engineering and Nutritional Science, Shaanxi Normal University, No. 620, \\ West Chang'an Avenue, Chang'an District, Xi'an 710119, Shaanxi, China; biniwang@snnu.edu.cn \\ * Correspondence: hejin04c@126.com (Y.H.); zhengjb@nwu.edu.cn (J.Z.); Tel.: +86-29-8825-8553 (Y.H.); \\ +86-29-8837-3025 (J.Z.); Fax: +86-29-8825-8553 (Y.H.); +86-29-8830-3448 (J.Z.)
}

Received: 29 August 2017; Accepted: 21 September 2017; Published: 27 September 2017

\begin{abstract}
Controllable preparation of nanopores to promote the performance of electrochemical biosensing interfaces has become one of the researching frontiers in biosensing. A double biocatalysis signal amplification of glucose biosensor for the study of electrochemical behaviors of glucose oxidase (GOx) was proposed by using horseradish peroxidase biosynthesized porous graphene (PGR) as the platform for the biocatalytic deposition of gold nanoparticles (AuNPs). The biosensor showed a linear range from 0.25 to $27.5 \mu \mathrm{M}$ with a detection limit of $0.05 \mu \mathrm{M}(\mathrm{S} / \mathrm{N}=3)$ towards glucose. Furthermore, the proposed AuNPs/GOx-PGR modified glassy carbon electrode (AuNPs/GOx-PGR/GCE) achieved direct electron transfer of GOx.
\end{abstract}

Keywords: biological amplification; glucose oxidase; horseradish peroxidase; porous graphene; Au nanoparticles

\section{Introduction}

Nanomaterials can be used to improve the catalytic performance, impart higher sensitivity, and enhance selectivity to an electrochemical measurement of analysts [1]. Different scales endow nanomaterials with regulable selectivity and sensitivity towards the electrochemical performance of crotonaldehyde, glucose, and electric methanol [2-4]. Especially, porous nanomaterials with loose structure can provide more reaction location for electrochemical reaction [5], endowing a better electrochemical response. It can react with atoms, ions, molecules, and nanoparticles on the surface and interior [6]. Additionally, the large surface area make it can load a lot of electroactive material and increase electron transfer rate. The existence of pore structure reduces the diffusion resistance of the electrolyte, which paves a smooth channel for the transference of ions and electrons [7]. Further, electrodes modified with porous nanomaterials change the distribution of the electric double layer on the interface. Effective performance improvement of electrochemical biosensing can be achieved by adjusting the pore structure and properties of porous nanomaterials [8]. Dergunov et al. controlled the particle behavior in nanopores by adjusting the $\mathrm{pH}$ value [9]. The investigation of electrochemical sensing behavior on porous nanogold surface indicated that [10] the special structure effectively improves the electrode direct electronic window, eliminate $\mathrm{pH}$ interferences, and promote biological activity. Proof-of-principle studies of DNA sequencing have been realized by a combination of engineered biological nanopores with polymer-based positional control [11]. Obviously, employment of nanopore structure in electrochemical sensing can improve its performance. Synthetic nanopores have tunable properties such as size, geometry, and surface chemistry, as well as enhanced mechanical, 
thermal, and chemical stability play an important role in the fabrication of novel sensing interface tunable property.

The good stability of carbon nanomaterials (CNMs) make themwidely applied to fabricate biosensors for electroanalytical investigations [12-14]. The relatively wide potential window, low background current, high catalytic properties, highly loading of biocatalysts, unique structure, and excellent electrical conductivity $[15,16]$ are beneficial for electrochemical processes. Graphene (GR) is a novel two-dimensional layered CNM with high specific surface area, preferable operability, and excellent electrical performance, and has been widely used in the fabrication of high-performance electrochemical sensors [17]. If some carbon atoms are removed from GR [18], a structure forms that generates pores with the edge of unsaturated carbon atoms, named as porous graphene (PGR). The PGR with specific structure tends to capture atoms and ions, forming a saturated bond. Furthermore, PGR can provide an active site for selective adsorption. Based on PGR, an electrochemical DNA migration sensing platform has been fabricated by researchers $[19,20]$. The results indicated that sensing performance could be adjusted by controlling the pore properties. Glucose oxidase (GOx) has been immobilized on PGR to realize direct electrochemistry and electrocatalysis with the significant advantages of sensitivity and linear range [21]. At present, electronic breakdown, microwave puffing technology, Pickering microemulsion method, and polystyrene template method [22-24] have been employed to prepare PGR. Pore structure on GR plane was generated by the internal oxidation induced by the catalysis of horseradish peroxidase (HRP) towards horseradish peroxidase [25]. The oxygen-containing groups on GR tend to close the heme center of HRP and other metal catalytic center enzymes, leading to a greater loading amount of the sensitive sensing film, which favors the selectivity and sensitivity of sensing. Compared with current carbon material modification methods for catalytic reaction [26-28], the modification of carbon nanomaterial by biocatalysis can be completed under moderate conditions, without heating, ultrasonicating, or strong acid or basic solutions. Moreover, the final nanocomplex by biocatalysis could have better catalytic ability for biosensing, due to the intrinsic interaction between enzymes and nanomaterials and accelerate electron transfer rate.

The aim of the present work is to controllably prepare PGR with versatile pore size by HRP-induced process, and then conduct in situ biocatalytic deposition of gold nanoparticles (AuNPs) onto the GOx-embedded PGR film to fabricate a novel biosensor for the determination of glucose level with the growth reaction. PGR is prepared by biocatalysis process of HRP and in situ biocatalytic deposition of AuNPs onto PGR by GOx. The two biocatalysts can play the role of double signal amplification to improve the sensitivity of the glucose biosensor. Herein, PGR can act not only as an enzyme immobilization reagent, but also as the biosensing platform for the determination of glucose level. Furthermore, the electrochemical behaviors of GOx at the proposed AuNPs/GOx-PGR/GCE (glassy carbon electrode) are also studied. It is expected that the proposed method has the advantage of design compatibility [29-32], which gives the electrochemical sensors the potential to be integrated for other devices and electronics.

\section{Experimental}

\subsection{Reagents}

GOx (E.C. 1.1.3.4, $182 \mathrm{Umg}^{-1}$, Type X-S from Aspergillusniger). Chloroauric acid tetrahydrate $\left(\mathrm{HAuCl}_{4} \cdot 4 \mathrm{H}_{2} \mathrm{O}\right)$ and $\mathrm{HRP}$ were purchased from Sigma (Los Angeles, CA, USA). High-purity graphite powder was purchased from the Shanghai Carbon Plant (Shanghai, China). Glucose stock solution was allowed to mutarotate at room temperature overnight before use. All other chemicals were of analytical reagent grade, and doubly distilled water was used in all the experiments. A $0.1 \mathrm{M}$ pH 7.0 sodium phosphate buffered saline (PBS) solution was used in all electrochemical studies unless otherwise stated. 


\subsection{Apparatus and Measurements}

A Jeol Neoscope Benchtop scanning electron microscope (SEM) was used for SEM images (Japan Electron Company, Tokyo, Japan). Transmission electron microscope (TEM) images were collected on an E.M. $912 \Omega$ energy-filtering TEM (120 kV) (Japan Electron Company, Tokyo, Japan). A DL-180 ultrasonic cleaning machine ( $35 \mathrm{KHz}$, Zhejiang Haitian Electron Instrument Factory, Kunshan, China) was used to dissolve and form a homogeneous solution. All electrochemical experiments were carried out on a CHI660e electrochemical workstation (Shanghai CH Instrument Co. Ltd., Shanghai, China) using a three electrode system. The working electrode was a GCE or a modified GCE. A saturated calomel electrode (SCE) and a platinum electrode served as reference and counter electrodes, respectively. All the electrochemical experiments were conducted at room temperature $\left(25 \pm 2{ }^{\circ} \mathrm{C}\right)$.

\subsection{Biosynthesized Porous Graphene}

First, $80 \mu \mathrm{L}$ of $5 \mathrm{mg} / \mathrm{mL}$ of HRP was added into $1 \mathrm{mg} / \mathrm{mL}, 2 \mathrm{mg} / \mathrm{mL}, 4 \mathrm{mg} / \mathrm{mL}$, and $6 \mathrm{mg} / \mathrm{mL}$ graphene oxide (GO) solutions and shaken in a rocking incubator at $37 \pm 0.2{ }^{\circ} \mathrm{C}$. Then, $20 \mu \mathrm{L} 3 \mathrm{M}$ $\mathrm{H}_{2} \mathrm{O}_{2}$ was added into the above solution, a $24 \mathrm{~h}$ interval passed, the addition of $\mathrm{H}_{2} \mathrm{O}_{2}$ was repeated and the operation was last for several days. The product was freeze-dried and stored in the refrigerator $\left(4^{\circ} \mathrm{C}\right)$ for later use.

\subsection{Preparation of GOx-Modified Electrode}

Before use, a glassy carbon electrode (GCE) of $3 \mathrm{~mm}$ diameter was polished to a mirror-like finish with 1.0, 0.3 , and $0.05 \mu \mathrm{m} \mathrm{Al}_{2} \mathrm{O}_{3}$ slurry on a polish cloth and rinsed with double-distilled water, then sonicated in ethanol and double-distilled water for $5 \mathrm{~min}$, respectively.

GOx-PGR composite was prepared by dispersing $1 \mathrm{mg}$ PGR and $5 \mathrm{mg}$ GOx into $1 \mathrm{~mL}$ PBS by shaking in a rocking incubator at $37 \pm 0.2^{\circ} \mathrm{C}$, mixing for $24 \mathrm{~h}$, then casting $10 \mu \mathrm{L}$ of the mixture onto the surface of the bare GCE by using a syringe to prepare GOx-PGR/GCE. Without use, the biosensor was stored at $4{ }^{\circ} \mathrm{C}$ in a refrigerator.

\subsection{Growth of $A u N P s$}

All growth solutions were purged with highly purified oxygen for $30 \mathrm{~min}$ before experiments, and an oxygen environment was kept over the solution during the growth process. The GOx-PGR/GCE was immersed into a growth solution consisting of $0.2 \mathrm{mM} \mathrm{HAuCl}_{4}$ in $0.1 \mathrm{M} \mathrm{PBS}$ with different concentrations of glucose for $25 \mathrm{~min}$, then it was removed from the above solution and transferred into 0.1 M PBS.

\section{Results and Discussion}

\subsection{The Controllable Biosynthesized Porous Graphene}

The pore structure on PGR was adjusted by reaction concentrations of GO and biocatalytic induction time. By comparison, 15 days was the optimal reaction time. At the beginning, there were only a few pores on GR. As the reaction time increased, the number of pore substantially increased. However, the amount of pores did not change further after 15 days. SEM images of products with different reaction concentrations of GO are shown in Figure 1 . When GO concentration was $1 \mathrm{mg} / \mathrm{mL}$, the pore structure was easily formed on GR with limited number. Increasing the concentration to $2 \mathrm{mg} / \mathrm{mL}$, the number of pores substantially increased with uniform pore size. Further increasing of GO concentration did not contribute to the increase of pore number. Further, the pore size was nonuniform, and the size became much larger-on the high order of micrometers. When GO concentration was increased to $6 \mathrm{mg} / \mathrm{mL}$, it was difficult to determine the pore structure on GR. All of the above indicates that the best condition for a biosynthesized pore structure on PGR was $2 \mathrm{mg} / \mathrm{mL} \mathrm{GO}$ reacted for 
15 days. TEM images of the product under optimal conditions are shown in Figure 2. It can be seen that the whole plane shows flake-like shape and nano-thickness GR with remarkable pore structure, as shown in Figure 2A. Pore structures with diameter 40 to $80 \mathrm{~nm}$ are labeled with a red line on the GR in Figure 2B. These pores played an important role in the further immobilization of GOx for biocatalytic growth of AuNPs on GR.
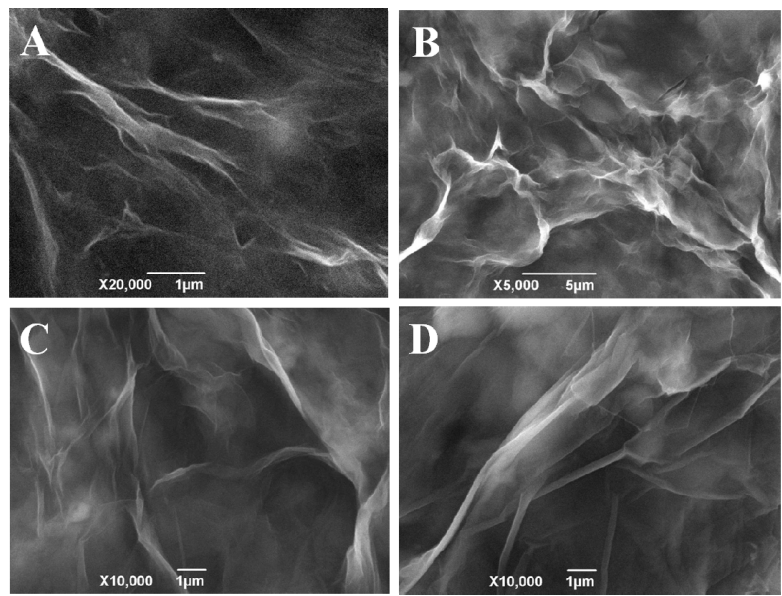

Figure 1. SEM images of product with different reaction concentrations of graphene oxide (GO) for 15 days: (A) $1 \mathrm{mg} / \mathrm{mL}$; (B) $2 \mathrm{mg} / \mathrm{mL}$; (C) $4 \mathrm{mg} / \mathrm{mL}$ and (D) $6 \mathrm{mg} / \mathrm{mL}$.
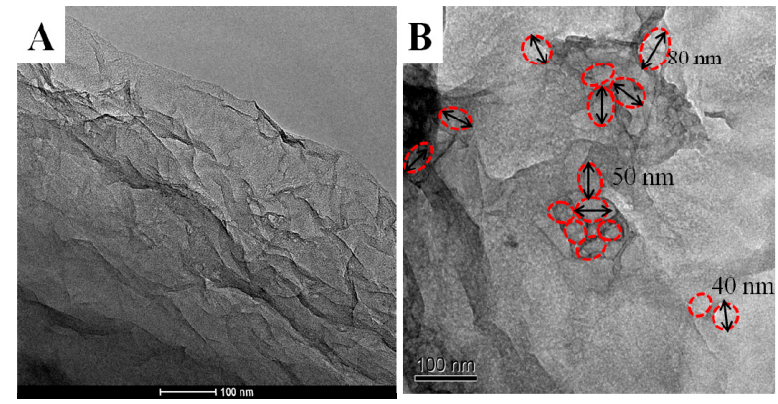

Figure 2. TEM images of product with reaction concentrations of GO $2 \mathrm{mg} / \mathrm{mL}$ for 15 days with low (A) and high order (B).

\subsection{The Mechanisms of Biocatalytically-Grown AuNPs}

The possible mechanism of the biocatalytic growth of AuNPs has been confirmed by our previous work $[33,34] . \mathrm{H}_{2} \mathrm{O}_{2}$ is produced by the oxidization of glucose from $\mathrm{GOx}$. Then, $\mathrm{H}_{2} \mathrm{O}_{2}$ reduces $\left[\mathrm{AuCl}_{4}\right]^{-}$ into $\mathrm{Au}(0)$, catalyzed by HRP on the surface of PGR. A double biocatalysis signal amplification was achieved for the determination of glucose. The sensitivity was greatly improved, with a value 1.2 to 1000 times higher than existing methods.

\subsection{Optimization of Sensor Performance}

The incubation time may affect the growth of AuNPs on the designed GOx-PGR/GCE. Subsequent experiments were carried out to obtain the optimum incubation time for GOx-induced AuNPs formation with a glucose concentration of $100 \mu \mathrm{M}$. The dependence of cyclic voltammetry (CV) peak current of the biocatalytic accumulation of AuNPs on accumulation time is displayed in Figure 3. The highest peak current was obtained when the accumulation time was $25 \mathrm{~min}$. As the reaction time increased, there was no significant increase of the peak current. Thus, the optimum accumulation time for the deposition of AuNPs was 25 min. Following experiments were carried out under this condition to get the best electrochemical performances. 


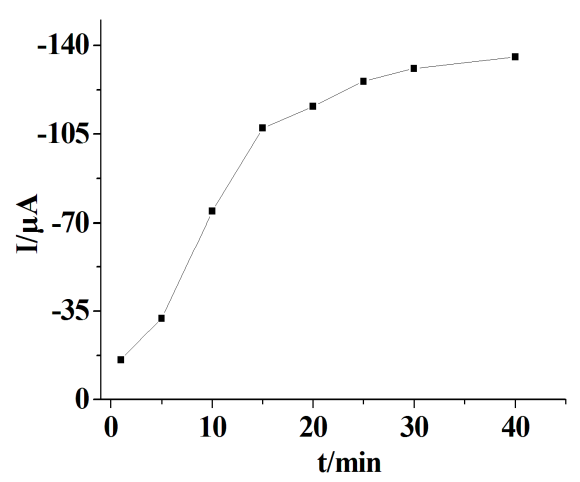

Figure 3. Plots of peak current vs. accumulation time.

\subsection{The Determination of Glucose Level}

Since the current response of AuNPs is related to the concentration of glucose, it can be used for the detection of glucose level. Figure 4 showed CVs on the GOx-PGR/GCE with increasing glucose concentration under the optimum conditions. After a preoxidation process before the detection, the reduction peak at a potential of about $0.4 \mathrm{~V}$ is weak due to $\mathrm{Au}(\mathrm{III})$, which is not beneficial for the sensitivity improvement. The oxidation peak of $\mathrm{Au}(\mathrm{III})$ at the potential of $0.85 \mathrm{~V}$ was chosen to describe the determination of glucose level, which was much smaller than our previous work. The current response at the potential of $0.85 \mathrm{~V}$ showed a linear relationship with the concentration of glucose. The potential was lower than our previous work [33,34], indicating a better anti-interference ability. The results showed a good linear relationship with the glucose concentration. The linear regression equation was $I_{s S}(\mu \mathrm{A})=-7.557 \mathrm{C}(\mu \mathrm{M})-75.31(r=0.9945)$ with linearity from 0.25 to $27.5 \mu \mathrm{M}$ (inset, Figure 4) and a detection limit of $0.05 \mu \mathrm{M}(\mathrm{S} / \mathrm{N}=3)$. The sensitivity was $755 \mu \mathrm{A} / \mathrm{mM}$. Compared with other methods, our biosensor showed sensitivity that was higher by 1.2 to 1000 times and a detection limit that was lower by one to three orders of magnitude. According to Michaelis-Menten kinetic mechanism, the apparent Michaelis-Menten constant $K_{M}^{a p p}$ value was calculated to be $67.4 \mu \mathrm{M}$, which was much lower than some previous reports. Herein, PGR offered a promising platform for glucose detection based on the GOx-induced formation of AuNPs. The method might be used to the detect blood sugar of humans in daily life with sweat or tears to intead the necessity of the blood collection of the existing test.

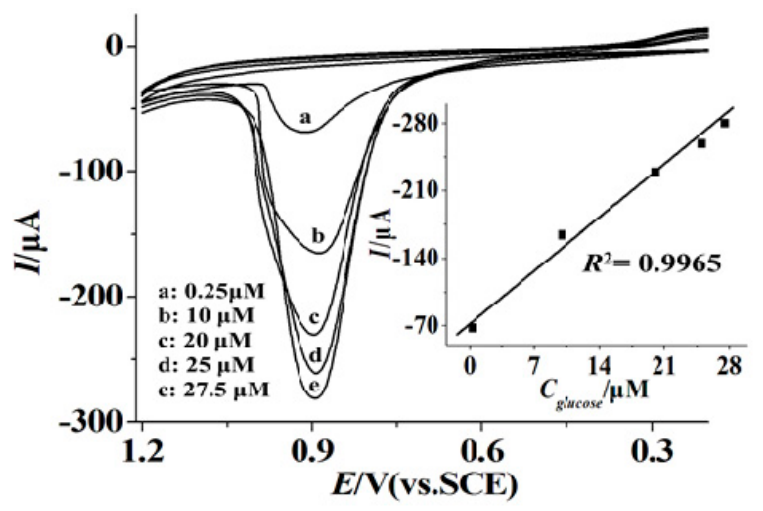

Figure 4. CVs of glucose oxidase (GOx)-induced formation of gold nanoparticles (AuNPs) for increasing concentrations of glucose. Glucose concentration ranges: (a) $0.25 \mu \mathrm{M}$; (b) $10 \mu \mathrm{M}$; (c) $20 \mu \mathrm{M}$; (d) $25 \mu \mathrm{M}$ and (e) $27.5 \mu \mathrm{M}$. Inset: plot of peak current vs. glucose concentration. Reaction solution: a stirred $0.1 \mathrm{M}$ phosphate buffered saline (PBS) solution containing $0.2 \mathrm{mM} \mathrm{HAuCl}_{4}$ and different concentrations of glucose. 


\subsection{Direct Electrochemistry}

CVs of the proposed AuNPs/GOx-PGR/GCE with different scan rates are shown in Figure 5. A pair of redox peaks with $E_{p a}=-0.217 \mathrm{~V}$ and $E_{p c}=-0.327 \mathrm{~V}$ (vs. SCE) are shown with the formal potential $E^{\circ \prime}=-0.272 \mathrm{~V}, \Delta E_{p}=90 \mathrm{mV}$ at the scan rate of $100 \mathrm{mV} / \mathrm{s}$. The ratio of cathodic to anodic current intensity is about 1 , indicating that a fast direct electron transfer reaction occurred. The value of $E^{\circ \prime}$ was close to the values reported previously [35,36]. According to the equation $I_{p}=n^{2} F^{2} v A \Gamma^{*} / 4 R T=n F Q v / 4 R T$ [37], the surface concentration of electroactive $\Gamma^{*}$ was calculated to be $2.34 \times 10^{-12} \mathrm{~mol} / \mathrm{cm}^{2}$, which matched the theoretical value $\left(2.86 \times 10^{-12} \mathrm{~mol} / \mathrm{cm}^{2}\right)$ for the monolayer of GOx on the bare electrode surface, indicating that the AuNPs/GOx-PGR/GCE keep the nanostructure of GR.

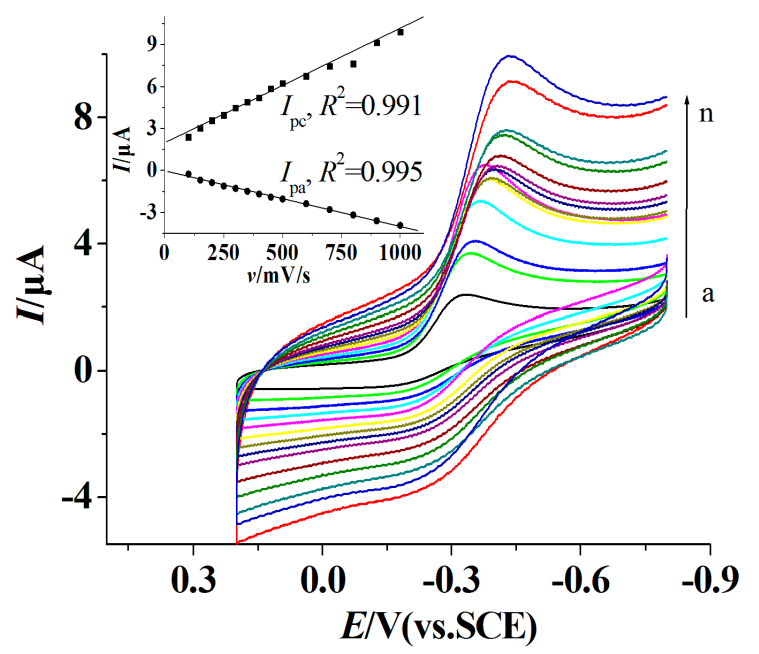

Figure 5. CVs of the AuNPs/GOx-PGR/GCE (glassy carbon electrode) in $\mathrm{N}_{2}$ saturated PBS with different scan rates (from a to $\mathrm{n}$ : 100, 150, 200, 250, 300, 350, 400, 450, 500, 600, 700, 800, 900, $1000 \mathrm{mV} / \mathrm{s}$ ).

\section{Conclusions}

In the present work, HRP-induced PGR with adjustable pore size was employed as the platform for in situ biocatalytic deposition of AuNPs, and a novel double biocatalysis (HRP+GOx) signal amplification glucose biosensor was constructed based on the CV response of AuNPs with different concentrations of glucose. The biosensor showed a low determination limit and high sensitivity for glucose detection. The reason can be ascribed to: (1) The PGR provided a benign microenvironment for the biocatalysis deposition process and the porous structure surface of PGR greatly enlarged the specific surface area to effectively increase the amount of GOx immobilized; (2) The advantages of biocatalysis itself, such as the accumulation and amplification effects; (3) Synergistic effects of PGR and AuNPs not only improved the electron transfer rate of the sensing system, but also exhibited the signal amplification of nanosize materials. Furthermore, the proposed AuNPs/GOx-PGR/GCE achieved the direct electrochemistry of GOx and electrocatalysis towards glucose. The present study may pave the way for the synthesis of novel nanomaterials with unique morphology and properties. Moreover, the advantage of the compatibility of the biosensor design can be integrated for other devices and electronics. Further works employing PGR as the biosensor platform are in our schedule.

Acknowledgments: The authors gratefully acknowledge the financial support of this project by the National Science Foundation of China (No. 21445004, 21545014, 21643014, 21275116), the Scientific Research Plan Projects of the Education Department of Shaanxi Province, China (No. 14JS095), Xi'an Science and Technology Plan Project (No. CXY1443WL27, CXY1531WL22, CXY1531WL34, 2016CXWL09), Key Disciplinary Areas of Analytical Chemistry \& the Industria Research Project of Science and Technology Department of Shaanxi Province (No. 2017GY-126); Startup funds from Xi'an University (No. 06005017) and the development transformation of key disciplines in Shaanxi provincial-analytical chemistry (No. 09009001). 
Author Contributions: For research articles with several authors, a short paragraph specifying their individual contributions must be provided. The following statements should be used "Yaping He and Jianbin Zheng conceived and designed the experiments; Yaping He and Bini Wang performed the experiments; Yaping He, Jianbin Zheng and Hongjiang Ren analyzed the data; Yaping He, Hongjiang Ren and Bini Wang contributed reagents/materials/analysis tools; Yaping He wrote the paper." Authorship must be limited to those who have contributed substantially to the work reported.

Conflicts of Interest: The authors declare no conflict of interest.

\section{References}

1. Chandran, G.; Li, X.; Ogata, A.; Penner, R.M. Electrically transduced sensors based on nanomaterials (2012-2016). Anal. Chem. 2017, 89, 249-275. [CrossRef] [PubMed]

2. Lizandara-Pueyo, C.; Siroky, S.; Wagner, M.R.; Hoffmann, A.; Reparaz, J.S.; Lehmann, M.; Polarz, S. Shape anisotropy influencing functional properties: Trigonal prismatic $\mathrm{ZnO}$ nanoparticles as an example. Adv. Funct. Mater. 2011, 21, 295-304. [CrossRef]

3. Rathod, D.; Dickinson, C.; Egan, D. Platinum nanoparticle decoration of carbon materials with applications in non-enzymatic glucose sensing. Sens. Actuators B Chem. 2010, 143, 547-554. [CrossRef]

4. Meng, H.; Xie, F.Y.; Chen, J. Morphology Controllable Growth of Pt Nanoparticles/nanowires on carbon powders and its application as novel electrocatalyst for methanol oxidation. Nanoscale 2011, 3, 5041-5048. [CrossRef] [PubMed]

5. Yang, G.; Yuan, R.; Chai, Y.Q. A high-sensitive amperometric hydrogen peroxide biosensor based on the immobilization of hemoglobin on gold colloid/1-cysteine/gold colloid/nanoparticles Pt-chitosan composite film-modified platinum disk electrode. Colloids Surf. B Biointerfaces 2008, 61, 93-100. [CrossRef] [PubMed]

6. Davis, M.E. Ordered Porous Materials for Emerging Applications. Nature 2002, 417, 813-821. [CrossRef] [PubMed]

7. Ding, L.X.; Wang, A.L.; Li, G.R.; Liu, Z.Q.; Zhao, W.X.; Su, C.Y.; Tong, Y.X. Porous Pt-Ni-P composite nanotube arrays: Highly electroactive and durable catalysts for methanol electrooxidation. J. Am. Chem. Soc. 2012, 134, 5730-5733. [CrossRef] [PubMed]

8. Bae, J.H.; Han, J.H.; Chung, T.D. Electrochemistry at nanoporous interfaces: New opportunity for electrocatalysis. Phys. Chem. Chem. Phys. 2012, 14, 448-463. [CrossRef] [PubMed]

9. Dergunov, S.A.; Durbin, J.; Pattanaik, S.; Pinkhassik, E. pH-Mediated catch and release of charged molecules with porous hollow nanocapsules. J. Am. Chem. Soc. 2014, 136, 2212-2215. [CrossRef] [PubMed]

10. Patel, J.; Radhakrishnan, L.; Zhao, B.; Uppalapati, B.; Daniels, R.C.; Ward, K.R.; Collinson, M.M. Electrochemical properties of nanostructured porous gold electrodes in biofouling solutions. Anal. Chem. 2013, 85, 11610-11618. [CrossRef] [PubMed]

11. Shi, W.; Friedman, A.K.; Baker, L.A. Nanopore sensing. Anal. Chem. 2017, 89, 157-188. [CrossRef] [PubMed]

12. Lu, X.B.; Xiao, Y.; Lei, Z.B.; Zhang, Q. A promising electrochemical biosensing platform based on graphitized ordered mesoporous carbon. J. Mater. Chem. 2009, 19, 4707-4714. [CrossRef]

13. Malhotra, R.; Papadimitrakopoulos, F.; Rusling, J.F. Sequential layer analysis of protein immunosensors based on single wall carbon nanotube forests. Langmuir 2010, 26, 15050. [CrossRef] [PubMed]

14. He, Y.P.; Sheng, Q.L.; Zheng, J.B.; Liu, B. Magnetite-Graphene for the direct electrochemistry of hemoglobin and its biosensing application. Electrochim. Acta 2011, 56, 2471-2476. [CrossRef]

15. Bertoncini, P.; Chauvet, O. Conformational Structural Changes of Bacteriorhodopsin Adsorbed onto Single-Walled Carbon Nanotubes. J. Phys. Chem. B 2010, 114, 4345-4350. [CrossRef] [PubMed]

16. Zhou, M.; Guo, J.D.; Guo, L.P.; Bai, J. Electrochemical sensing platform based on the highly ordered mesoporous carbon-fullerene system. Anal. Chem. 2008, 80, 4642-4650. [CrossRef] [PubMed]

17. Wu, S.X.; He, Q.Y.; Tan, C.L.; Zhang, H. Graphene-Based electrochemical sensors. Small 2013, 9, $1160-1172$. [CrossRef] [PubMed]

18. Qiu, W.Z.; Nguyen, P.; Skafidas, E. Graphene nanopores: Electronic transport properties and design methodology. Phys. Chem. Chem. Phys. 2014, 16, 1451-1459. [CrossRef] [PubMed]

19. Merchant, C.A.; Healy, K.; Wanunu, M.; Ray, V.; Peterman, N.; Bartel, J.; Fischbein, M.D.; Venta, K.; Luo, Z.T.; Johnson, A.T.; et al. DNA translocation through graphene nanopores. Nano Lett. 2010, 10, $2915-2921$. [CrossRef] [PubMed] 
20. Siwy, Z.S.; Davenport, M. Nanopores: Graphene opens up to DNA. Nat. Nanotechnol. 2010, 5, 697-698. [CrossRef] [PubMed]

21. Wang, Y.; Li, H.X.; Kong, J.L. Facile Preparation of mesocellular graphene foam for direct glucose oxidase electrochemistry and sensitive glucose sensing. Sens. Actuators B Chem. 2014, 193, 708-714. [CrossRef]

22. Kim, T.Y.; Jung, G.; Yoo, S.; Kwang, S.S.; Rodney, S.R. Activated graphene-based carbons as supercapacitor electrodes with macro- and mesopores. ACS Nano 2013, 7, 6899-6905. [CrossRef] [PubMed]

23. Gu, X.Y.; Ning, Y.; Yang, Y.; Wang, C.Y. One-Step synthesis of porous graphene-based hydrogels containing oil droplets for drug delivery. RSC Adv. 2014, 4, 3211-3218. [CrossRef]

24. Wilson, P.; Mbah, G.N.; Smith, T.G.; Schmidt, D.; Lai, R.Y.; Hofmann, T.; Sinitskii, A. Three-Dimensional periodic graphene nanostructures. J. Mater. Chem. C 2014, 2, 1751-1752. [CrossRef]

25. Gregg, P.K.; Brett, L.A.; Harindra, V.; Yanamala, N.; Kapralov, A.A.; Tyurina, Y.Y.; Klein-Seetharaman, J.; Kagan, V.E.; Star, A. The enzymatic oxidation of graphene oxide. ACS Nano 2011, 5, 2098-2108.

26. Liu, T.; Yu, K.; Gao, L.; Chen, H.; Wang, N.; Hao, L.H.; Li, T.X.; He, H.C.; Guo, Z.H. Graphene quantum dots decorated $\mathrm{SrRuO}_{3}$ mesoporous film as an efficient counter electrode for high-performance dye-sensitized solar cells. J. Mater. Chem. A 2017, 5, 17848-17855. [CrossRef]

27. Liu, Z.; Huang, Z.; Cheng, F.; Guo, Z.H.; Wang, G.; Chen, X.; Wang, Z. Efficient dual-site carbon monoxide electro-catalysts via interfacial nano-engineering. Sci. Rep. 2016, 6, 33127-33137. [CrossRef] [PubMed]

28. Wang, Y.; He, Q.; Wei, H.; Guo, J.; Ding, K.Q.; Wang, Q.; Wei, S.Y.; Guo, Z.H. Optimal Electrocatalytic $\mathrm{Pd} / \mathrm{MWNTs}$ nanocatalysts toward formic acid oxidation. Electrochim. Acta 2015, 184, 452-465. [CrossRef]

29. Alippi, C. A unique timely moment for embedding intelligence in applications. CAAI Trans. Intell. Technol. 2016, 1, 1-3. [CrossRef]

30. Jin, H.Y.; Chen, Q.; Chen, Z.X.; Hu, Y.; Zhang, J.W. Multi-Leap motion sensor based demonstration for robotic refine tabletop object manipulation task. CAAI Trans. Intell. Technol. 2016, 1, 104-113. [CrossRef]

31. Zhang, X.Y.; Gao, H.B.; Guo, M.; Li, G.P.; Liu, Y.C.; Li, D.Y. A study on key technologies of unmanned driving. CAAI Trans. Intell. Technol. 2016, 1, 4-13. [CrossRef]

32. Padhy, S.; Panda, S. A hybrid stochastic fractal search and pattern search technique based cascade PI-PD controller for automatic generation control of multi-source power systems in presence of plug in electric vehicles. CAAI Trans. Intell. Technol. 2017, 2, 12-25. [CrossRef]

33. Zheng, J.B.; He, Y.P.; Sheng, Q.L.; Zhang, H.F. DNA as a linker for biocatalytic deposition of Au nanoparticles on graphene and its application in glucose detection. J. Mater. Chem. 2011, 21, 12873-12879. [CrossRef]

34. Zhang, H.F.; Liu, R.X.; Sheng, Q.L.; Zheng, J.B. Enzymatic deposition of Au nanoparticles on the designed electrode surface and its application in glucose detection. Colloids Surf. B 2011, 82, 532-535. [CrossRef] [PubMed]

35. Li, K.T.; Liu, B.; Zheng, J.B.; Sheng, Q.L.; Liu, R.X. Direct electrochemistry of glucose oxidase on nail-like carbon and its biosensing for glucose. Electroanalysis 2010, 22, 701-706. [CrossRef]

36. Liu, J.P.; Guo, C.X.; Li, C.M.; Li, Y.Y.; Chi, Q.B.; Huang, X.T.; Liao, L.; Yu, T. Carbon-decorated ZnO nanowire array: A novel platform for direct electrochemistry of enzymes and biosensing applications. Electrochem. Commun. 2009, 11, 202-205. [CrossRef]

37. Laviron, E. The use of linear potential sweep voltammetry and of a.c. voltammetry for the study of the surface electrochemical reaction of strongly adsorbed systems and of redox modified electrodes. J. Electroanal. Chem. Interfacial Electrochem. 1979, 100, 263-270. [CrossRef]

(C) 2017 by the authors. Licensee MDPI, Basel, Switzerland. This article is an open access article distributed under the terms and conditions of the Creative Commons Attribution (CC BY) license (http:/ / creativecommons.org/licenses/by/4.0/). 